\title{
The Pseudomonas aeruginosa alternative sigma factor PvdS controls exotoxin A expression and is expressed in lung infections associated with cystic fibrosis
}

\author{
Tracey A. Hunt, † Wen-Tao Peng, Isabelle Loubens and Douglas G. Storey
}

Author for correspondence: Douglas G. Storey. Tel: +1 403220 5274. Fax: +1 4032989311.

e-mail: storey@ucalgary.ca

Department of Biological Sciences, University of Calgary, 2500 University Dr. NW, Calgary, Alberta, Canada T2N 1N4

\begin{abstract}
PvdS is an alternative sigma factor regulated by the global iron regulator Fur. It has been demonstrated that PvdS plays a role in the iron-dependent regulation of exotoxin A (ETA) in Pseudomonas aeruginosa strain PA01. The goals of this research were to determine if pvdS was transcribed by the bacteria in the chronic lung infections associated with cystic fibrosis (CF) and to determine how PvdS interacts with the regAB promoters of the hypertoxigenic strain PA103. It was found that pvdS is transcribed in the lungs of patients with CF and that it appears to be involved with the regulation of toxA in this environment. This correlated with the finding that in strain PA103, a mutation in pvdS reduced ETA activity while the same mutation in strain PAO1 abrogated ETA production. It was also shown that in strain PA103, pvdS was absolutely required for activation of the regAB P2 promoter. The effect of PvdS on the P2 promoter may be direct or indirect; however, in support of a direct role, an eight-out-of-nine base-pair match to the consensus sequence for PvdS binding was identified at the transcriptional start site for the P2 promoter. The effect of PvdS on the PA103 regAB P1 promoter under aerobic growth conditions was also examined. The results show that PvdS does modulate the expression from this promoter but that both the regAB operon and PvdS are required for optimal $\mathrm{P1}$ promoter activity. These studies demonstrate that the alternative sigma factor PvdS acts as a regulator of ETA expression in $P$. aeruginosa strain PA103 through the regAB operon and that PvdS is expressed in lung infections associated with CF.
\end{abstract}

Keywords: RegA, pvdS, toxA, iron regulation, strain PA103

\section{INTRODUCTION}

Pseudomonas aeruginosa is an opportunistic pathogen that causes extensive morbidity and mortality in individuals who are immunocompromised or have underlying medical conditions such as cystic fibrosis (CF) (Bodey et al., 1983). The virulence of $P$. aeruginosa during an infection is multi-factorial and includes the production of many virulence factors, one of the most toxic of which is exotoxin A (ETA) (Liu, 1974). ETA is

\footnotetext{
† Present address: Dept of Microbiology and Immunology, University of Western Ontario, London, Ontario, Canada.

Abbreviations: CF, cystic fibrosis; ETA, exotoxin A.
}

an ADP-ribosylating toxin that acts on eukaryotic elongation factor II to inhibit protein synthesis within the target host cell (Iglewski \& Kabat, 1975). The regulation of ETA expression at the level of transcription is complex and involves a cascade of regulators that result in the maximal production of ETA in ironlimiting environments (Grant \& Vasil, 1986; reviewed by Vasil \& Ochsner, 1999). In high-iron conditions $(100 \mu \mathrm{M})$, tox $A$ transcripts cannot be detected, while in low-iron conditions, toxA mRNA can be identified during the late-exponential and stationary phases of growth (Lory, 1986).

Many regulators have been speculated to act on the toxA gene, but RegA is believed to act directly on the tox $A$ promoter. RegA is a positive regulatory protein 
that is required for the specific enhancement of ETA synthesis (Hedström et al., 1986). ETA expression was demonstrated to be completely abrogated in the $\operatorname{reg} A B$ knockout strain PA103AregAB::Gm, confirming that the $\operatorname{reg} A B$ locus is required for ETA production (Raivio et al., 1996). Because RegA is required for ETA production, the environmental regulation of tox $A$ expression is believed to be mediated through the $\operatorname{reg} A B$ operon.

The regulation of the reg $A B$ operon is strain dependent. Strain PAO1, the prototypical strain, when grown under aerobic conditions, transcribes only $\operatorname{reg} A$, and primary transcription occurs from an iron-regulated promoter designated P2 (Wick et al., 1990). PAO1 is missing the start codon of the regB ORF (Wick et al., 1990). In the hypertoxigenic strain PA103, the regAB operon is preceded by two independent promoters that regulate the transcription of separate transcripts (Frank et al., 1989; Storey et al., 1990). The P1 promoter is located 164 bp upstream from the translational start site of $\mathrm{reg} A$ and is not tightly regulated by iron. The $\mathrm{P} 2$ promoter is active only in low-iron conditions and is located 75 nucleotides upstream of the $\operatorname{reg} A$ start codon. In the hypertoxigenic strain PA103, when grown under aerobic conditions, it has been demonstrated that a functional $\operatorname{reg} B$ gene is required in order for transcription from the $\mathrm{P} 1$ promoter to occur but activity from the $\mathrm{P} 2$ promoter is independent of $r e g B$ (Wick et al., 1990). Interestingly, it has been shown that when strain PAO1 is grown under microaerobic conditions, both $\mathrm{P} 1$ and $\mathrm{P} 2$ promoters are active (Ochsner et al., 1996; Barton et al., 1996). These observations further demonstrate the complexity of the regulation and the importance of environmental effects.

To date, at least seven regulators have been implicated in regulation of the $\operatorname{reg} A B$ promoters: $\operatorname{reg} B$ (Wick et al., 1990; Storey et al., 1991), PtxR (Hamood et al., 1996), PtxS (Swanson et al., 1999), Vfr (West et al., 1994; Albus et al., 1997), LasR (Gambello et al., 1993), Fur (Prince et al., 1991) and PvdS (Ochsner et al., 1996). Of these regulatory elements, only $\mathrm{PvdS}$ appears to be involved in the regulation of both promoters in strain PAO1 (Ochsner et al., 1996). pvdS was originally identified as a regulatory gene involved in activating pyoverdine synthesis (Cunliffe et al., 1995; Miyazaki et al., 1995). The PvdS (pyoverdine sigma factor) protein shows homology to Escherichia coli FecI, an alternative sigma factor. Based on this strong homology, and the presence of a putative DNA-binding motif at the C-terminal end, $\mathrm{PvdS}$ was postulated to act as an alternative sigma factor for RNA polymerase (Cunliffe et al., 1995; Miyazaki et al., 1995). The PvdS protein from P. aeruginosa has been overexpressed and purified (Leoni et al., 2000). The protein binds to the promoter regions of $p v d A, p v d E$ and $p v d F$, and acts as an alternative sigma factor with $E$. coli RNA polymerase to initiate transcription of $p v d A$ (Wilson \& Lamont, 2000; Wilson et al., 2001). PvdS expression is repressed by iron and this regulation is mediated through the global iron regulator Fur (Ochsner et al., 1995). PvdS plays a key role in iron regulation in
$P$. aeruginosa and has been demonstrated to be involved in the regulation of pyoverdine biosynthesis genes and ETA production (Miyazaki et al., 1995; Leoni et al., 1996; Ochsner et al., 1996). Recently, Lamont et al. (2002) have shown that PvdS may also be controlled by an anti-sigma factor FpvR. Ochsner et al. (1996) have also demonstrated that in the prototypical strain PAO1, PvdS was necessary for $\operatorname{reg} A$ expression. Their data suggested that $\mathrm{PvdS}$ interacts either directly or indirectly with the P2 promoter of $\operatorname{reg} A$ (Ochsner et al., 1996; reviewed by Vasil \& Ochsner, 1999). PvdS may modulate expression from the $\operatorname{reg} A \mathrm{P} 1$ promoter but is not required for activity in strain PAO1. On a functional basis, $p v d S$ has been recently demonstrated to be required for optimal virulence in an infective endocarditis model (Xiong et al., 2000). The role of PvdS in the pathogenesis of $P$. aeruginosa may, therefore, be more important than previously considered.

In this study, we wanted to establish whether $p v d S$ is expressed and involved in the regulation of ETA during the chronic lung infections associated with the genetic disease CF. Our second objective was to determine if PvdS plays a role in the regulation of the $\operatorname{reg} A B$ operon in strain PA103, since in PA103 the operon appears to be regulated differently from the $r e g A$ gene in strain PAO1.

\section{METHODS}

Media. E. coli cultures were grown in Luria-Bertani broth (LB; Sambrook et al., 1989) and P. aeruginosa cultures were grown in trypticase soy broth dialysate chelexed (TSBDC; Liu, 1973) as previously described. For maintenance of plasmids in E. coli, antibiotics were added to a final concentration of $100 \mu \mathrm{g}$ ampicillin $\mathrm{ml}^{-1}, 15 \mu \mathrm{g}$ gentamicin ml ${ }^{-1}$ or $50 \mu \mathrm{g}$ streptomycin $\mathrm{ml}^{-1}$. For growth of $P$. aeruginosa strains containing plasmids or chromosomal insertions, media contained antibiotics at final concentrations of $400 \mu \mathrm{g}$ carbenicillin $\mathrm{ml}^{-1}, 300 \mu \mathrm{g}$ gentamicin $\mathrm{ml}^{-1}$ or $500 \mu \mathrm{g}$ streptomycin $\mathrm{ml}^{-1}$. All antibiotics were purchased from Sigma. In liquid TSBDC media, high-iron conditions were achieved by adding $\mathrm{FeCl}_{3}$ (Sigma) to a final concentration of $10 \mu \mathrm{g} \mathrm{ml}^{-1}$. Low-iron conditions were achieved by acid washing all glassware with $20 \% \mathrm{HCl}$ before use. Wherever possible, sterile iron-free plastic equipment was used in place of glassware.

Bacterial strains and plasmids. Bacterial strains and plasmids used in this study are listed in Table 1 . The strain $P$. aeruginos IL-1 was constructed by the gene replacement systems reported by Quandt \& Hynes (1993) and Schweizer (1992). To construct the gene replacement plasmid, a $6 \mathrm{~kb}$ Pst I-SmaI fragment from a genomic clone of $\operatorname{reg} A$ was ligated into pUC12. A $3 \cdot 2 \mathrm{kbp}$ BamHI fragment from pZ1918 (Schweizer, 1993 b) containing a promoterless lacZ gene was then ligated into the first BamHI site of the regA ORF. A sacRB gene from pMH1801 (Hynes et al., 1989) was digested with PstI and the fragment containing the $s a c R B$ gene was ligated into the PstI site. The resulting plasmid was electroporated into $P$. aeruginosa PA103 and recombinants were selected for as previously described (Quandt \& Hynes, 1993; Schweizer, 1992). The recombinant clone IL-1 was used in this study. The reporter construct prevents transcription from the P1 promoter because a functional $\operatorname{reg} B$ is required for P1 activity in strain PA103 and $\operatorname{reg} A B$ is not transcribed from IL-1. Transcription unique to the $\mathrm{P} 2$ promoter of the $\operatorname{reg} A B$ operon 
Table 1. Bacterial strains and plasmids

\begin{tabular}{|c|c|c|}
\hline Strain or plasmid & Description & Reference \\
\hline \multicolumn{3}{|l|}{ P. aeruginosa } \\
\hline PA103 & $\operatorname{reg} A^{+} \operatorname{reg} B^{+}$hypertoxigenic lab. strain & Liu (1966) \\
\hline PAO1 & $\operatorname{reg} A^{+} \operatorname{reg} B$ prototrophic lab. strain & Holloway et al. (1979) \\
\hline IL-1 & PA103 regA::lacZ; P2-lacZ $\mathrm{P}^{-}$phenotype & This study \\
\hline PA103 pvdS:: Gm & Gm cassette at the $S t u \mathrm{I}$ site of the $p v d S$ gene in strain PA103; $\mathrm{Gm}^{\mathrm{R}}$ & This study \\
\hline $\mathrm{PAO} 1 \Delta p v d S$ & $\begin{array}{l}\text { PAO1 with a } 460 \text { bp deletion in the } p v d S \text { gene; Gm cassette at the } p v d S \\
\text { locus; } \mathrm{Gm}^{\mathrm{R}}\end{array}$ & Ochsner et al. (1996) \\
\hline IL-1 $p v d S:: \Omega$ & $\Omega$ cassette at the $S t u \mathrm{I}$ site of the $p v d S$ gene in strain IL-1; Sm ${ }^{\mathrm{R}} / \mathrm{Spec}^{\mathrm{R}}$ & This study \\
\hline \multicolumn{3}{|l|}{ Plasmids } \\
\hline pUC181.8 & $\begin{array}{l}\text { pUC18 containing the } 1.8 \mathrm{~kb} \text { Pst I stabilizing fragment }(\mathrm{SF}) \text { for Pseudomonas } \\
\text { replication; } \mathrm{Cb}^{\mathrm{R}}\end{array}$ & Frank \& Iglewski (1988) \\
\hline $\mathrm{pTH} p v d S$ & $\begin{array}{l}1005 \text { bp pvdS PCR product ligated in the EcoRI site of pUC181.8; } \mathrm{Cb}^{\mathrm{R}}(p v d S \\
\text { is under the control of its native promoter) }\end{array}$ & This study \\
\hline pDF191.8-202 & $\begin{array}{l}\text { pUC19 containing the } 1 \cdot 8 \text { Pst I fragment from pRO1614 and the regAB operon } \\
\text { from strain PA103 (the } \operatorname{reg} A B \text { operon is under the control of its native } \\
\text { promoters) }\end{array}$ & Frank et al. (1989) \\
\hline pUCGm & $\begin{array}{l}\text { Plasmid containing the Tn1696-derived } \mathrm{Gm}^{\mathrm{R}} \text { cassette flanked by the pUC19 } \\
\text { MCS; Gm }\end{array}$ & Schweizer (1993a) \\
\hline $\mathrm{pHP} 45 \Omega$ & $1.9 \mathrm{~kb} \mathrm{Sm}^{\mathrm{R}} / \mathrm{Spec}^{\mathrm{R}}$ interposon cassette in $\mathrm{pHP} 45 ; \mathrm{Ap}^{\mathrm{R}} \mathrm{Sm}^{\mathrm{R}} / \mathrm{Spec}^{\mathrm{R}}$ & Prentki \& Krisch (1984) \\
\hline pJQ200SK & Suicide vector containing the $s a c B$ cassette; pBluescript II SK MCS; $\mathrm{Gm}^{\mathrm{R}}$ & Quandt \& Hynes (1993) \\
\hline $\mathrm{pJQ} p v d S:: \mathrm{Gm}$ & $\begin{array}{l}\text { Gm ligated into the } S t u I \text { site of } p v d S ; p v d S:: \text { Gm ligated into the XhoI and } \\
\text { XbaI sites of pJQ200SK; } \mathrm{Gm}^{\mathrm{R}}\end{array}$ & This study \\
\hline $\mathrm{pJQ} p v d S:: \Omega$ & $\begin{array}{l}\Omega \text { ligated into the } S t u \mathrm{I} \text { site of } p v d S ; p v d S:: \Omega \text { ligated into the XhoI and XbaI } \\
\text { sites of pJQ200SK; } \mathrm{Sm}^{\mathrm{R}} / \mathrm{Spec}^{\mathrm{R}}\end{array}$ & This study \\
\hline
\end{tabular}

can be monitored in this strain by performing $\beta$-galactosidase assays.

Plasmid pTHpvdS was constructed from the 1005 bp PCR product of the $p v d S$ locus from $P$. aeruginosa strain PA103 (forward primer AAG AAT TCC TCC CCT CCA TCA TTC GCA G; reverse primer: ACG AAT TCT ACC TGG ACG GTC CCG TTG). The PCR product was flanked by EcoRI sites to aid in cloning. Sequence analysis confirmed that this PCR product contained the complete $563 \mathrm{bp} p v d S$ gene, the 148 bp promoter region, and 294 bp of flanking chromosomal DNA. Plasmid pTHpvdS contains the entire $p v d S$ ORF and expression is mediated from its native promoter. This plasmid was used to study the effect of $p v d S$ expression from a multicopy plasmid, but under the regulatory control of its native iron-regulated promoter.

In order to investigate the effects of interrupting the $p v d S$ gene on the chromosome of strains IL-1 and PA103, knock-out strains were constructed. In knock-outs of PA103 and IL-1, the $p v d S$ ORF was interrupted with an $\Omega$ cassette from pHP45 $\Omega$ or a Gm cassette from pUCGm respectively, at the unique $S t u \mathrm{I}$ site within the $p v d S$ ORF. The interrupted $p v d S$ gene was constructed on a suicide plasmid that contained a $s a c B$ cassette (pJQ200SK) to facilitate the double crossover onto the chromosome. The resulting plasmids, pJQpvdS:: $\Omega$ and $\mathrm{pJQ} p v d S:: \mathrm{Gm}$, were electroporated into $P$. aeruginosa strains IL-1 and PA103 to produce strains IL-1 pvdS:: $\Omega$ and PA103 pvdS:: Gm respectively.

DNA manipulations. Plasmid DNA was isolated based on the alkaline lysis method developed by Birnboim \& Doly (1979). Chromosomal DNA was isolated from P. aeruginosa using a modified version of the method described by Ausubel et al.
(1991). All restriction enzymes and buffers used were purchased from Gibco-BRL or Pharmacia. Electroporations were performed on $P$. aeruginosa following the method described by Smith \& Iglewski (1989).

Southern hybridization. Southern blotting was performed as described by Ausubel et al. (1991) with some modifications. DNA fragments to be used as probes were labelled with $\left[\gamma^{32} \mathrm{P}\right] \mathrm{dCTP}$ (Dupont NEN Products) using an Oligolabelling Kit (Pharmacia).

Growth curve methodology. ADP-ribosyltransferase and $\beta$ galactosidase assays were performed on culture samples collected over the course of a growth curve. Growth curves were performed over a 24-30 h time period and samples were collected at the time points indicated. All growth curves were repeated at least twice. Samples for $\beta$-galactosidase assays were collected and stored at $-80^{\circ} \mathrm{C}$ until $\beta$-galactosidase assays were performed on each sample in duplicate using a modification of the method of Miller (1972). Samples of cellfree supernatant were collected and stored at $-80^{\circ} \mathrm{C}$ until extracellular ADP-ribosyltransferase activity was assayed in triplicate for each sample as described by Chung \& Collier (1977).

Analysis of RNA from bacterial populations found in sputa. The patient population analysed was as previously described by Storey et al. (1998). All patients and their guardians gave their consent to participate in this study. The study design was ethically reviewed and approved by the Conjoint Research Ethics Board at the University of Calgary. All techniques used in the collection, handling and extraction of the samples were as previously described by Storey et al. (1992). The DNA probe for $p v d S$ was a $1 \mathrm{~kb} E c o$ RI fragment from pTHpvdS 
containing the $p v d S$ gene. Probes for tox $A$ and $\operatorname{alg} D$ were prepared as described by Storey et al. (1997). Statistical analysis was as described by Storey et al. (1998).

\section{RESULTS}

\section{Transcription of $p v d S$ in the lungs of patients with CF}

We have previously shown that both $\operatorname{reg} A B$ transcripts are produced in the lungs of patients with CF (Raivio et al., 1994). We have also demonstrated that toxA is transcribed in the CF lung (Storey et al., 1992). It was, therefore, of interest to determine if $p v d S$ is transcribed in the lungs of CF patients, and whether or not there was a correlation between the transcript accumulation of $p v d S, \operatorname{reg} A B$ and toxA. Fig. 1 shows that we were able to detect $p v d S$ transcripts at levels above background in $80 \%$ of our CF samples. There is a range of transcript accumulation from background levels to greater than 20 times the optimal transcription when cells are grown in the laboratory (see sample 24 in Fig. 1b). These results represent 16 samples in which the RNA was extracted from equivalent numbers of bacteria and equivalent amounts of RNA were loaded on the slot blot. The variation in expression levels also suggested that $p v d S$ is regulated in the lungs of patients with CF. We hypothesize that $p v d S$ may be regulating the $\operatorname{reg} A B$ operon and subsequently toxA. We would have liked to correlate pudS expression to the $\operatorname{reg} A B$ operon since pvdS directly regulates this operon. However, the halflife of the two $\operatorname{reg} A$ transcripts is very short and, therefore, they are difficult to detect in sputa (Raivio et al., 1994). Thus, we have compared the transcript accumulation of toxA to $p v d S$ since it appears that $p v d S$ indirectly regulates toxA. A Spearman rank correlation was performed on the 16 samples shown in Fig. 1. This analysis showed a statistically significant correlation between $p v d S$ and toxA transcript accumulation $(r=0.647, P<0.005)$. Notably, we did not detect a statistically significant correlation between either $\operatorname{alg} D$ and $p v d S(r=0.430 P<0.05)$ or $\operatorname{alg} D$ and toxA $(r=0.385$ $P<0 \cdot 1)$ transcript accumulation in these samples, as expected. Overall this analysis suggests that PvdS is an important regulator of ETA in the lungs of patients with CF. Raivio et al. (1996) showed that the pattern of $\operatorname{reg} A B$ transcript accumulation in the lungs of patients with $\mathrm{CF}$ was similar to the pattern found in the hypertoxigenic strain PA103 when growing under aerobic conditions. This led us to question if the role of PvdS in strain PA103 is identical to or different from its role in the regulation of the prototypical strain PAO1.

\section{$P$. aeruginosa $p v d S$ knock-out strains produce less ETA than the parental strain PA103}

Initially we wanted to determine if a mutation in $p v d S$ affected ETA production in the hypertoxigenic strain PA103 in the same way as in the prototypical strain PAO1. Fig. 2 shows the ETA production for the pvdS mutants and their complemented controls over $28 \mathrm{~h}$ of growth. Strain PA103 pvdS::Gm produced approximately $18-25 \%$ of the maximum ETA produced by the complemented strain (Fig. 2a). Interestingly, the ETA produced by PA103 pvdS::Gm remains tightly iron
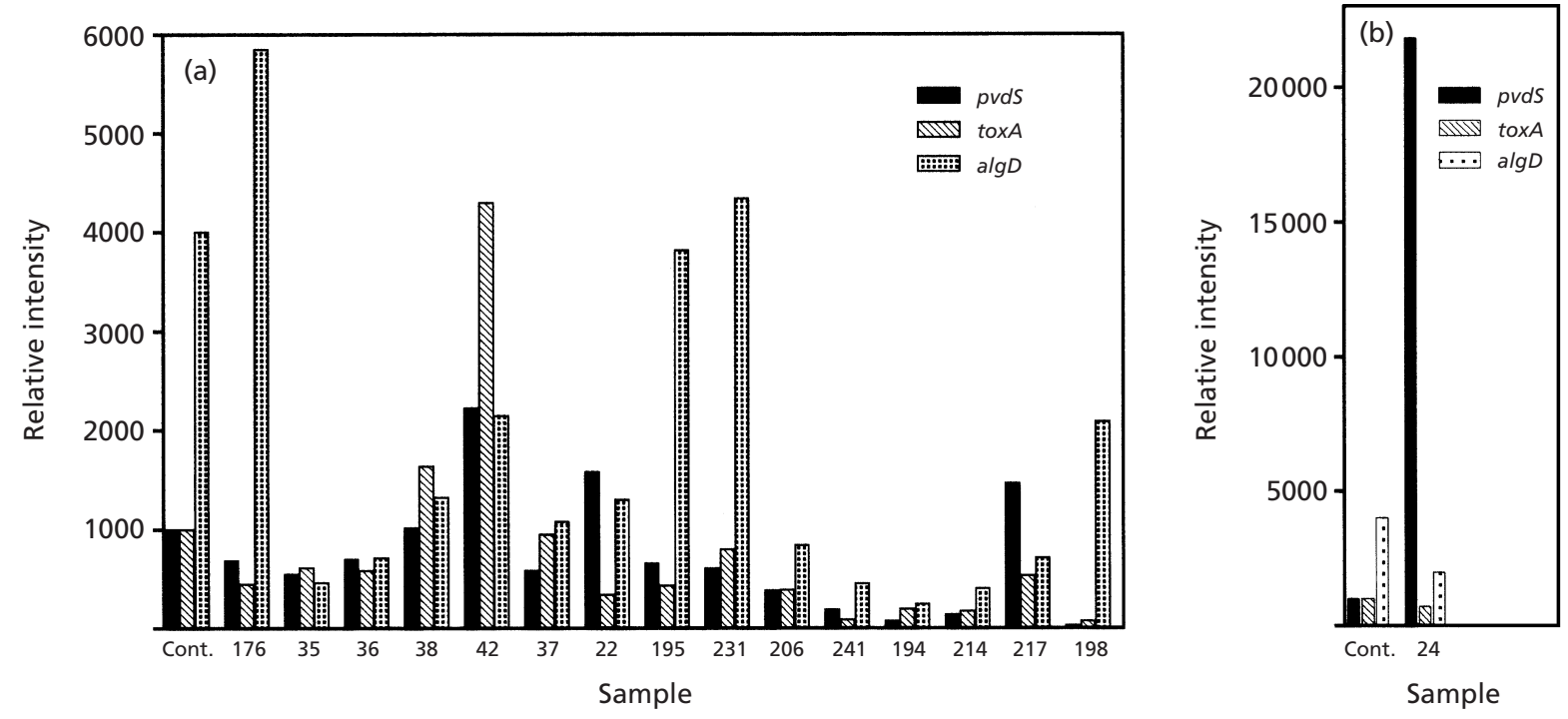

Fig. 1. PvdS is transcribed in the lungs of patients with $C F$ : population transcript accumulation of $P$. aeruginosa pvdS, tox $A$ and $a l g D$ in RNA extracted from CF sputa. All samples contained equivalent numbers of $P$. aeruginosa $\left(10^{8} \mathrm{ml}^{-1}\right)$ and in all samples $P$. aeruginosa was the only pathogenic bacterium isolated. A soft laser scanning densitometer was utilized to determine the relative intensity from an autoradiograph of the blot. The results represent the signal from $10 \mu \mathrm{g}$ RNA extracted from an equivalent number of bacteria. Panels (a) and (b) represent the results from one experiment set. Panel (b) was separated to reflect the much higher values for sample no. 24. Control hybridizations (cont.) are identical for both panels. 


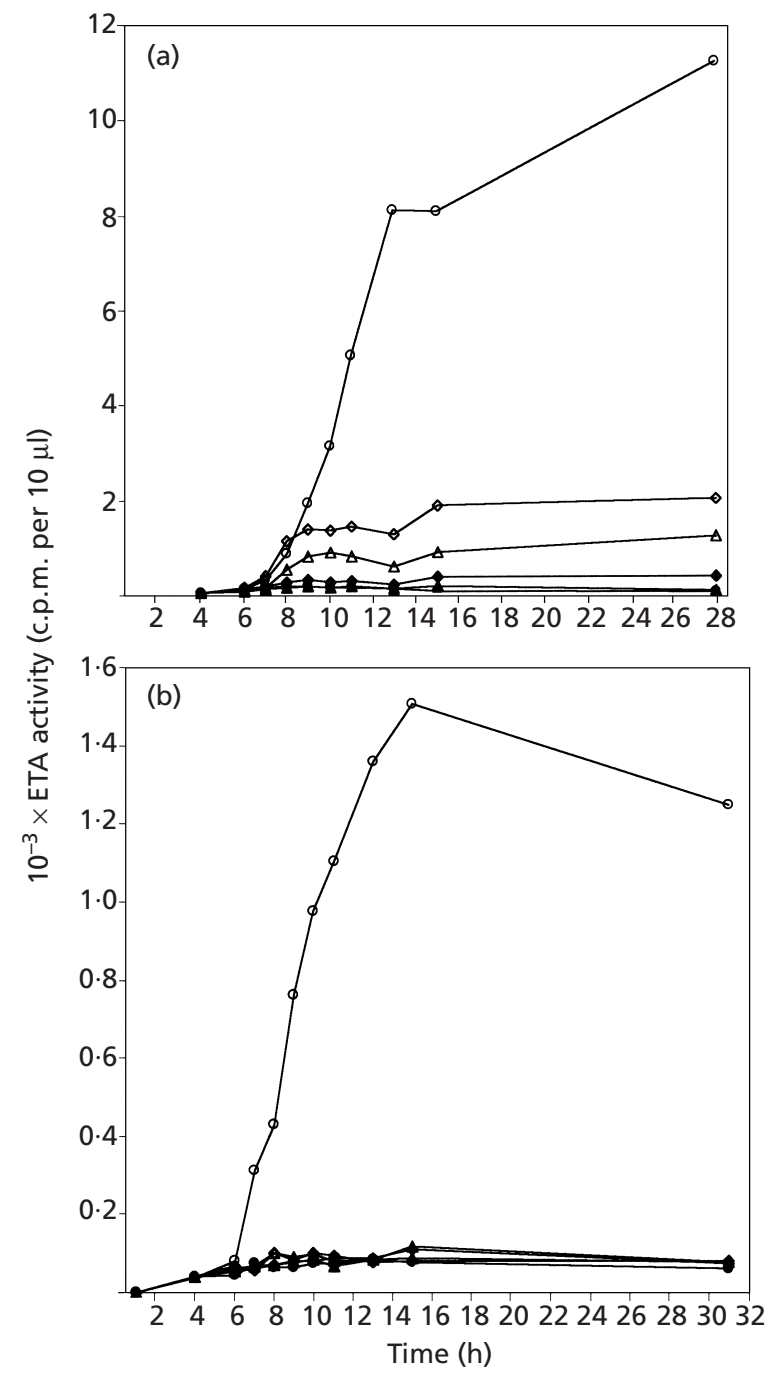

Fig. 2. PvdS is required for optimal ETA production in pvdS knockouts of PA103 and PAO1. Cultures were grown for $28 \mathrm{~h}$ under high-iron conditions $\left(10 \mu \mathrm{g} \mathrm{ml}^{-1}\right.$; black symbols) or ironlimiting conditions (open symbols) and aliquots were removed at the indicated time points. Extracellular ADP-ribosyltransferase activity was assayed in triplicate at each time point. (a) ETA production in a pvdS knockout of the hypertoxigenic strain PA103. PA103 pvdS::Gm $(\diamond, \diamond)$, PA103 pvdS::Gm with the vector control pUC181.1 ( $\Delta, \triangle)$, and PA103 pvdS::Gm with the plasmid pTHpvdS $(\bullet, \bigcirc)$. (b) PAO1 $\Delta p v d S(\diamond, \diamond)$, PAO1 $\Delta p v d S$ with the vector control pUC181.1 $(\Delta, \triangle)$, and PAO1 $\Delta p v d S:: G m$ with the plasmid pTHpvdS $(\bullet, \bigcirc)$.

regulated (Fig. 2a). These results suggest that $p v d S$ is required for maximal ETA production in PA103; however a mutation in $p v d S$ does not abrogate the iron regulation of the residual ETA activity. In contrast, in strain PAO1 the $p v d S$ mutation completely abrogates ETA activity, as previously shown by Ochsner et al. (1996) (Fig. 2b). In this experiment the residual ETA activity in PAO $1 \Delta p v d S$ ( $1 \%$ of maximal ETA activity in the complemented strain) was not affected by the iron concentration of the medium. Thus, it appears that there are significant differences in the effect that a mutation in

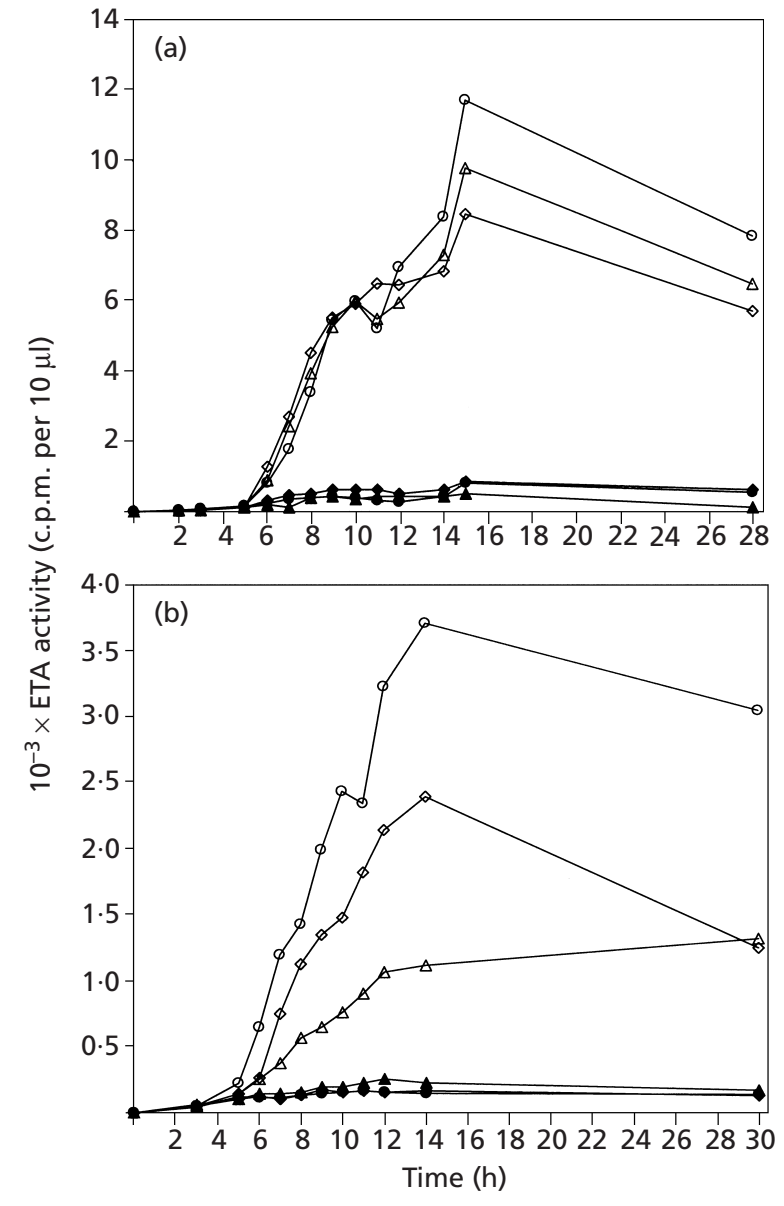

Fig. 3. ETA activity is increased under iron-limiting conditions in the presence of multi-copy pvdS in strain PA103 and strain PAO1. Cultures grown under high-iron conditions $\left(10 \mu \mathrm{g} \mathrm{ml}^{-1}\right)$ are indicated by black symbols; cultures grown under ironlimiting conditions are indicated by open symbols. (a) PA103 $(\diamond, \diamond)$, PA103 with the vector control pUC181.1 $(\mathbf{\Delta}, \triangle)$, and PA103 with the plasmid pTHpvdS, expressing pvdS under the control of the native promoter $(\bullet, \bigcirc)$. (b) PAO1 $(\diamond, \diamond)$, PAO1 with the vector control pUC181.1 $(\boldsymbol{\Lambda}, \triangle)$, and PAO1 with the plasmid pTHpvdS, expressing pvdS under the control of the native promoter $(\bullet, \bigcirc)$.

pvdS has on the ETA regulation of PA103 as compared to the prototypical PAO1.

\section{ETA production is increased when PvdS is expressed on a multi-copy plasmid in low-iron conditions}

pvdS expression is controlled by the iron-regulated repressor Fur (Barton et al., 1996; Ochsner et al., 1996). It therefore was of interest to determine if multiple copies of pvdS would enhance the expression of ETA in either high- or low-iron media. Plasmids pUC181.8 and pTHpvdS were electroporated into the hypertoxigenic strain PA103. Growth of the strains was observed over $28 \mathrm{~h}$. In all cases no growth differences were observed between the strains; however growth was slower in lowiron conditions than in high-iron conditions (data not shown). The results in Fig. 3(a) demonstrate that when 


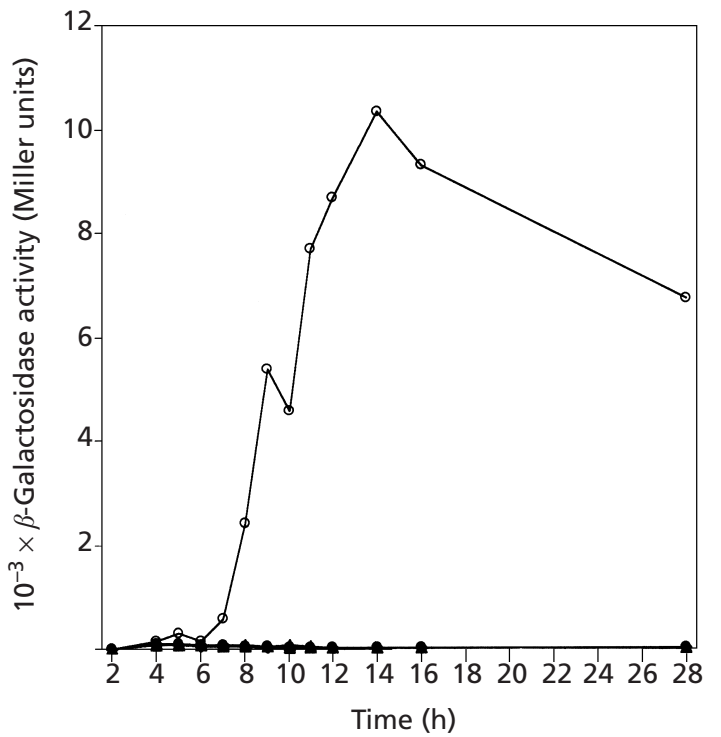

Fig. 4. The reduced $P 2$ activity from strain $I L-1 p v d S:: \Omega$ is restored in low-iron conditions when the mutation is complemented in trans with pvdS. Strain IL-1 contains a chromosomal P2-lacZ fusion. The six cultures were grown for $28 \mathrm{~h}$ and aliquots were assayed for $\beta$-galactosidase activity in duplicate at each indicated time point. Cultures grown under high-iron conditions $\left(10 \mu \mathrm{g} \mathrm{ml}^{-1}\right)$ are indicated by black symbols; cultures grown under iron-limiting conditions are indicated by open symbols. $\diamond, \diamond, \mathrm{IL}-1$ pvdS:: $\Omega ; \mathbf{\Lambda}, \triangle, \mathrm{IL}-1$ pvdS:: $\Omega$ with the vector control pUC181.1;,$\bigcirc$, IL-1 pvdS:: $\Omega$ with the plasmid pTHpvdS.

$p v d S$ is provided in trans, ETA production of strain PA103 is increased by approximately $16-27 \%$ in lowiron conditions. Enhanced ETA production solely in low-iron conditions demonstrates that multiple copies of the $p v d S$ promoter are not sufficient to dilute out the iron-bound Fur at high iron concentrations.

In strain PAO1, multiple copies of $p v d S$ also did not affect ETA production in high-iron conditions (Fig. 3b). However, in low-iron conditions we observed a more dramatic effect in this strain, with multiple copies of pvdS giving a $35-70 \%$ increase in maximal ETA production (Fig. 3b) as compared to strain PA103, which showed only a $16-27 \%$ increase. Thus, it appears that multiple copies of pvdS have different effects on ETA regulation in PAO1 as compared to PA103.

\section{Influence of PvdS on regA P2 activity when $P$. aeruginosa strain PA103 is grown in low-iron conditions}

Previous research in strain PAO1 (Ochsner et al., 1996), and the effect on ETA production that we observed in PA103pvdS:: Gm and in PA103 with multiple copies of pvdS (Fig. 2a and 3a) suggested that PvdS may be interacting differently with the PA103 regA promoters than with the $\operatorname{reg} A$ promoters of PAO1. In order to monitor activity from the $\mathrm{P} 2$ promoter of the $\operatorname{reg} A B$ operon, strain IL-1 was constructed from the parental strain PA103. P. aeruginosa strain IL-1 contains a lacZ reporter gene insertion within the $\operatorname{reg} A B$ ORF integrated into the chromosome. The reporter construct in IL-1 lacks transcription from the P1 promoter because a functional $\operatorname{reg} B$ is required for P1 activity (Wick et al., 1990) and $\operatorname{reg} A B$ is not transcribed in this strain. Therefore, activity from the $\mathrm{P} 2$ promoter of the $\operatorname{reg} A B$ operon can be examined in this strain by monitoring the expression from the chromosomal lac $Z$ reporter gene. To examine the effect of $p v d S$ on the P2 promoter, we generated a mutant of IL-1 containing a $p v d S$ knockout. No P2 activity was detected in either high- or lowiron conditions from strain IL-1 $p v d S:: \Omega$ (Fig. 4). However, P2 activity from this knock-out strain was restored to parental levels when complemented with $p v d S$ and grown under iron-limiting conditions. Notably, the expression of the P2 promoter remains highly regulated in the complemented strain, as P2 activity is evident only during the late-exponential and early stationary phases of growth under iron-limiting conditions. These results suggest that $\mathrm{PvdS}$ is required for the iron-regulated activity from the $\mathrm{P} 2$ promoter of the regAB operon in PA103. A similar finding was shown for the P2 promoter in PAO1 (Ochsner et al., 1996). Together these findings support the hypothesis that the alternative sigma factor PvdS acts as a regulator of the $\mathrm{P} 2$ promoter of the $\operatorname{reg} A B$ operon, and that $\mathrm{PvdS}$ may directly bind the $\operatorname{reg} A B \mathrm{P} 2$ promoter.

\section{The P2 promoter of the regAB operon may contain a consensus sequence for PvdS binding}

The sequence upstream of the $\operatorname{reg} A B$ operon was analysed for homologies to known iron-regulatory consensus sequences. In the $\operatorname{reg} A B$ promoter region we identified an eight-out-of-nine base-pair match (Table 2) to the published PvdS consensus sequence (Rombel et al., 1995; Wilson et al., 2001). This partial consensus sequence is located between -77 and -68 from the translational start site for the RegA protein and between -1 and +8 of the transcriptional start site for the T2 transcript. The one mismatched base was in the centre of the sequence with a $G$ instead of an $A$. The sequence in the $\mathrm{P} 2$ promoter region was compared to known and putative PvdS binding sequences (Table 2). The central adenine was conserved in all the other binding sites (Table 2). Another difference between the RegA binding site and the others is that in the RegA regulatory sequence the putative PvdS binding site sits directly over the transcriptional start site. All the other binding sites appear to be located upstream of the respective transcriptional start sites. It should be noted that $p v d A, E$ and $F$ have been demonstrated to be regulated by $\mathrm{PvdS}$ (Leoni et al., 1996, 2000; Wilson \& Lamont, 2000). The other promoters in Table 2, including the $\operatorname{reg} A$ promoter, have not been examined with respect to $p v d S$ regulation. The identification of this partial PvdS consensus binding sequence in the $\mathrm{P} 2$ promoter region suggests that PvdS may be a regulator of the ironregulated P2 promoter of the $\operatorname{reg} A B$ operon, and 
Table 2. Promoter region of PvdS-regulated genes

\begin{tabular}{|c|c|c|}
\hline Iron-regulated promoter* & Binding sequence $\dagger$ & $\begin{array}{c}\text { Base-pair } \\
\text { match }\end{array}$ \\
\hline P. aeruginosa $p v d A$ promoter ${ }^{1,2}$ & ${ }^{-38}$ CTTAAATTC ${ }^{-30}$ & $8 / 9$ \\
\hline P. aeruginosa pvdD promoter ${ }^{2,3}$ & ${ }^{-39} \mathrm{GCTAAATCC}{ }^{-29}$ & $8 / 9$ \\
\hline P. aeruginosa pvdE promoter $^{2,3}$ & ${ }^{-23} \mathrm{GCTAAATAC}^{-15}$ & $9 / 9$ \\
\hline P. aeruginosa pvdF promoter $^{2}$ & ${ }^{-38} \mathrm{CGTAAATTG}^{-30}$ & $9 / 9$ \\
\hline$P$. aeruginosa tox $A$ promoter ${ }^{4}$ & ${ }^{-62} \mathrm{CATAAATCC} \mathrm{C}^{-54}$ & $7 / 9$ \\
\hline P. putida siderophore promoter ${ }^{4}$ & ${ }^{-75} \mathrm{CCTAAATCC}{ }^{-67}$ & $8 / 9$ \\
\hline P. sp. strain M114 iron-regulated promoter ${ }^{4}$ & ${ }^{-91} \mathbf{A C T A A T T C C} \mathrm{C}^{-83 x}$ & $6 / 9$ \\
\hline P. aeruginosa reg $A B$ promoter $\mathrm{P} 2^{5}$ & ${ }^{-1} \mathrm{GCTAGATAC} \mathrm{C}^{+8}$ & $8 / 9$ \\
\hline Consensus PvdS binding sequence & $(\mathrm{G} / \mathrm{C})(\mathrm{G} / \mathrm{C}) \operatorname{TAAAT}(\mathrm{T} / \mathrm{A})(\mathrm{C} / \mathrm{G})$ & $9 / 9$ \\
\hline
\end{tabular}

* The consensus sequence similarity was identified by: 1, Miyazaki et al. (1995); 2, Wilson et al. (2001); 3, Merriman et al. (1995); 4, Rombel et al. (1995); 5, this work.

† Nucleotide positions are indicated with respect to the predicted transcriptional start site except $\left({ }^{x}\right)$, which is in relation to the translational start site. Nucleotides that differ from the consensus are shown in bold.

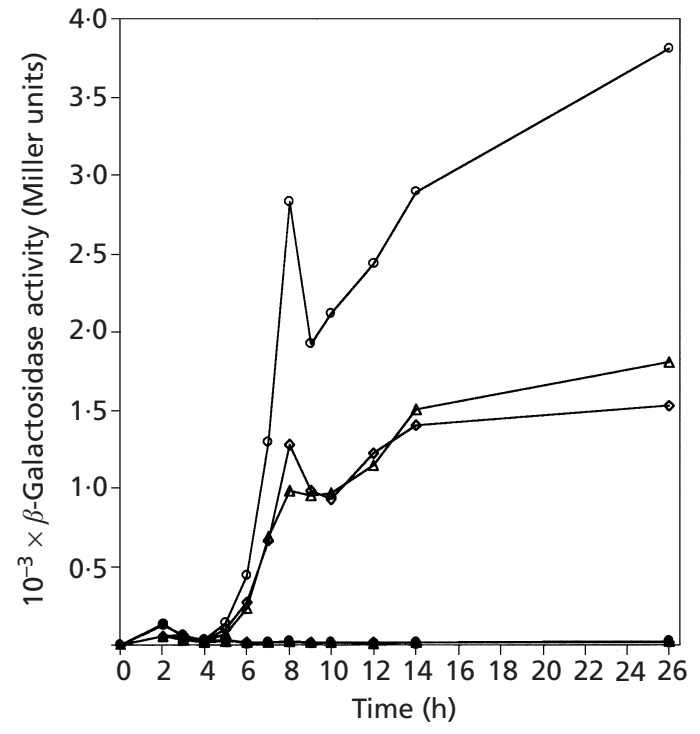

Fig. 5. Activity from the $P 2$ promoter is increased in the presence of multiple copies of pvdS under iron-limiting conditions. Cultures grown under high-iron conditions $(10 \mu \mathrm{g}$ $\mathrm{ml}^{-1}$ ) are indicated by black symbols; cultures grown under iron-limiting conditions are indicated by open symbols. $\diamond, \diamond$, $\mathrm{IL}-1 ; \Delta, \triangle$ IL-1 with the vector control pUC181.1; $, \mathrm{O}, \mathrm{IL}-1$ with the plasmid pTHpvdS, overexpressing pvdS.

therefore an indirect regulator of ETA production in $P$. aeruginosa.

\section{Activity from the P2 promoter of regAB is increased when PvdS is expressed from a multi-copy plasmid with the native promoter}

The results in Fig. 3(a) revealed that multiple copies of $p v d S$ enhance ETA production but that this production remained iron regulated. Our hypothesis is that in strain
PA103, PvdS may be acting on the P2 promoter to regulate expression of the $\operatorname{reg} A B$ operon. To examine the effect of multiple copies of $p v d S$ on the PA103 reg $A B$ P2 promoter, plasmids pUC181.8 and pTHpvdS were electroporated into strain IL-1 and P2 promoter activity was monitored over $26 \mathrm{~h}$ of growth. The results (Fig. 5) demonstrate that after growth for 14-24 h with pvdS expressed from a multi-copy plasmid, P2 activity is increased by a factor of $93-111 \%$. Furthermore, this enhancement in P2 activity is dependent on iron concentration, indicating that iron regulation is maintained. Thus it appears that the effect of multiple copies of $p v d S$ on the regAB P2 promoter is very similar to the effect on production of ETA, supporting a regulatory link between PvdS and toxA occurring through RegA.

\section{The toxA promoter is not regulated by PvdS independently of RegA}

An eight-out-of-nine base-pair match to the PvdS consensus binding sequence has previously been identified in the toxA promoter region (Table 2) (Rombel et al., 1995; Wilson et al., 2001). Because of the existence of this potential binding sequence, we examined whether PvdS could regulate the production of ETA directly at the toxA promoter in addition to regulating ETA through the P2 promoter of $\operatorname{reg} A B$. In order to examine the possible role of PvdS regulation at the toxA promoter, extracellular ETA production was assayed from $P$. aeruginosa strain IL-1. Strain IL-1 possesses a functional tox $A$ locus but an interrupted $\operatorname{reg} A B$, so any ETA produced from this strain is independent of the transcriptional activator RegA. ETA production was assayed from strains IL-1, IL-1(pUC181.8) and IL1 (pTHpvdS) in both high- and low-iron TSBDC and these assays confirmed that only negligible amounts of ADP-ribosyltransferase activity were observed from all three strains in both high- and low-iron conditions (data not shown). This indicates that PvdS cannot activate the 

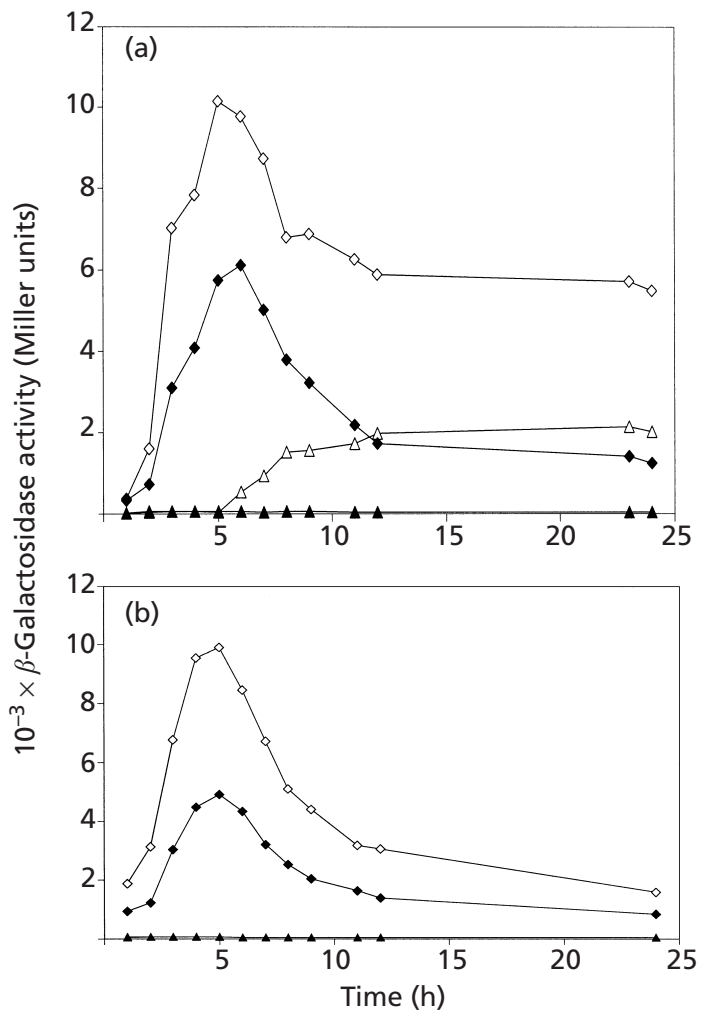

Fig. 6. Effect of PvdS on the regAB promoters. The cultures were grown in low-iron medium (a) or high-iron medium (b) for $24 \mathrm{~h}$ and aliquots were assayed for $\beta$-galactosidase activity in duplicate at each time point. $\triangle$, IL-1 with the vector control pUC181.8; $\Delta$, IL-1 pvdS:: $\Omega$ with the vector control pUC181.8; $\diamond$, IL-1 complemented with the regAB operon on pDF191.8$202 ; \diamond, \mathrm{IL}-1 \mathrm{pvdS}:: \Omega$ complemented with the regAB operon on pDF191.8-202.

toxA promoter independently of the transcriptional activator RegA and that the regulation of ETA production by this alternative sigma factor may be indirect through the $\operatorname{reg} A B$ operon.

\section{Interaction of PvdS with the P1 promoter of regAB in strain PA103}

A critical difference in $\operatorname{reg} A$ regulation between strains PA103 and PAO1 is that when cells are grown under aerobic conditions, the $\mathrm{P} 1$ promoter of $\operatorname{reg} A$ is active in PA103 whereas in PAO1 it is inactive. In strain PA103, a requirement for $\mathrm{P} 1$ activity is the presence of a functional regAB operon. To examine the effect of PvdS on the P1 promoter, we introduced the $\operatorname{reg} A B$ operon in trans on plasmid pDF191.8-202 into strains IL-1 and IL-1 $p v d S:: \Omega$ because the chromosomal copy of the $\operatorname{reg} A B$ operon in these strains is inactivated by an insertion of lacZ. The results in Fig. 6(a) demonstrate that expression of $\operatorname{reg} A B$ from a multi-copy plasmid dramatically increases the early activity from the chromosomal reg $A B$ operon in both IL-1 and IL-1 $p v d S:: \Omega$, suggestive of P1 activity. During stationary phase, the levels of $\beta$-galactosidase activity in IL-1 $p v d S:: \Omega$ (pDF191.8-202) drop to below those of IL1 (pDF191.8-202), suggesting that the P2 promoter is not active in strain IL-1 $p v d S:: \Omega$ (pDF191.8-202) when the $\operatorname{reg} A B$ operon is expressed in trans. Fig. 6(a) also shows that in the $\operatorname{reg} A B$-complemented strains, the $\mathrm{P} 1$ activity is greater in strain IL-1 than in strain IL-1 $p v d S:: \Omega$ at both early and late stages of growth. This suggests that activity from the P1 promoter may be modulated by PvdS but that it is not required. To confirm these results, we repeated these experiments in high-iron conditions, where the $\operatorname{reg} A B \mathrm{P} 1$ promoter is active but the $\mathrm{P} 2$ promoter is repressed. The results in Fig. 6(b) show that when the strains are grown in high-iron conditions, early expression is observed in the strains complemented by the reg $A B$ operon in trans. Furthermore, levels were lower in the $p v d S$ knock-out strain, further supporting the hypothesis that $\mathrm{PvdS}$ is not required for P1 activity but may modulate it. As expected, in both complemented strains, there is a decrease in activity in stationary phase, indicating that the $\mathrm{P} 2$ promoter is not active when the strains are grown under high-iron conditions. Northern blot analysis of the parental strains PA103 and PA103 $p v d S:: \Omega$ also demonstrates that PvdS may modulate transcription from the P1 promoter and it is essential for transcription from the $\mathrm{P} 2$ promoter (data not shown). This is a clear difference from the previously described strain PAO1, where the P1 promoter is not active under these conditions.

It is interesting that the early P1 activity in a strain with an active $p v d S$ is unaffected by the iron concentration of the medium. In the $p v d S$ knock-out strain, there is a slight iron effect, with the culture growing in high iron having about $20 \%$ less activity than the culture growing in low iron. Overall, our data suggest that PvdS has a general modulatory effect on the P1 promoter. Interestingly, we could not detect a putative binding site in the area of the regAB P1 promoter, suggesting that $\mathrm{PvdS}$ may act indirectly on this promoter, probably through an additional regulatory element.

\section{DISCUSSION}

PvdS is an alternative sigma factor for RNA polymerase. This classification was originally based on protein homologies to known alternative sigma factors and the presence of a putative DNA-binding motif at the Cterminal end of the protein (Cunliffe et al., 1995; Miyazaki et al., 1995). P. aeruginosa PvdS binds to E. coli core RNA polymerase and to $p v d A$ promoter DNA (Leoni et al., 2000; Wilson \& Lamont, 2000). The PvdS-core RNA polymerase complex also directs transcription of the pyoverdine biosynthesis genes $p v d E$ and $p v d F$ (Wilson et al., 2001). Furthermore, it has been demonstrated that PvdS expression is regulated by iron through the global iron repressor Fur (Ochsner et al., 1996). PvdS is transcribed only in low-iron conditions; in high-iron conditions transcription is repressed by Fur. In this study, we examined the expression of $p v d S$ by $P$. aeruginosa during the chronic lung infections associated 
with $\mathrm{CF}$, and the role of this alternative sigma factor in the regulation of toxA in both the hypertoxigenic strain PA103 and the prototypical strain PAO1.

Recently, the oxygen- and iron-dependent sigma factor PvdS has been shown to be important in an infective endocarditis model (Xiong et al., 2000). What is less clear is the role that PvdS may play in human infections. In some infections, such as the chronic lung infections associated with the genetic disease CF, expression of PvdS could be used as a marker for specific environmental conditions. In these infections, it appears that iron is limiting, as siderophores have been detected in the sputa of these patients (Haas et al., 1991). However, the oxygen concentration that bacteria are exposed to in the lungs of patients with CF has not been determined. It is likely that over the course of these chronic infections the bacterial populations are exposed to varying oxygen levels. Also, it is likely that within the environment of the lung, different bacterial populations would be exposed to varying levels of oxygenation. With these possibilities in mind, we have extracted RNA from the total bacterial populations found in sputa from CF patients and analysed the sputa for transcription of pvdS. These samples were solely from paediatric patients, where it might be expected to see considerable variation in their lung disease and, hence, variation in the oxygen levels. We demonstrated that in samples with equivalent numbers of bacteria and containing only $P$. aeruginosa, there were varying levels of $p v d S$ transcript accumulation (from background levels to 20 times the control levels of transcript accumulation; Fig. 1). This variability suggests that the bacteria are exposed to different concentrations of iron and/or oxygen within these samples. The results also suggest that the levels of pvdS transcription in the lungs of CF patients is being regulated. We then analysed the transcript accumulation patterns of $p v d S$, toxA and algD. Even though we analysed a relatively small sample size $(n=16)$, we were able to detect a significant correlation between $p v d S$ transcript accumulation and toxA transcript accumulation. In contrast, we were not able to show a significant correlation between $p v d S$ transcript accumulation and algD transcript accumulation. Taken together, our data suggest that $p v d S$ is transcribed in the lungs of patients with CF and that PvdS may at least be partially responsible for the regulation of toxA within the lungs of CF patients. Since $p v d S$ is repressed by anoxic conditions, our data also suggest that the bacterial population is exposed to a reasonable level of oxygen in some samples.

We also examined the functional role of PvdS in strain PA103. This strain was chosen because its ETA regulatory pattern seems to match that of $P$. aeruginosa in the lungs of individuals with CF (Raivio et al., 1994). Further, PA103 differs from the prototypical strain PAO1 in the regulation of ETA production. The $p v d S$ knockout strain PA103pvdS:: Gm produced only $20 \%$ of the ETA produced by the complemented strain (Fig. 2a). This result was different from the findings in strain PAO1, where ETA production was totally abrogated in strain PAO1 $1 p v d S$ (Fig. 2b) (Ochsner et al., 1996). The tighter control by PvdS in strain PAO1 was also observed in experiments where $p v d S$ was introduced into PAO1 and PA103 on a multi-copy vector. These experiments revealed a greater percentage increase in ETA activity in PAO1 than that in PA103 (Fig. 3). This difference is probably due to some residual RegA production from the $\operatorname{reg} A B$ promoters in strain PA103.

Ochsner et al. (1996) showed that PvdS was essential for the iron regulation of the $\operatorname{reg} A \mathrm{P} 2$ promoter in strain PAO1. When the pudS gene of strain PA103 was disrupted, activity from the P2 promoter was not detected, irrespective of the iron conditions (Fig. 4). P2 promoter activity was only observed from this knockout strain when $p v d S$ was expressed in trans under ironlimiting conditions. Likewise, when $p v d S$ was introduced on a multi-copy vector in to the parental PA103 strain, regA P2 activity was enhanced (Fig. 5). These studies suggest that in strain PA103, as in strain PAO1, $\mathrm{PvdS}$ is essential for $\operatorname{reg} A \mathrm{P} 2$ promoter activity. Interestingly, these functional studies are supported by the discovery of a putative PvdS binding site in the P2 promoter region of the $\operatorname{reg} A B$ operon (Table 2).

The regulation of the $\operatorname{reg} A \mathrm{P} 1$ promoter was also analysed in strain PA103 to determine if it might account for the differences in ETA activity between strains PA103 and PAO1. In strain PAO1, the P1 promoter is not active under aerobic conditions (Ochsner et al., 1996). Here we show that in strain PA103 growing under aerobic conditions, $\mathrm{P} 1$ promoter activity is modulated by the presence of $p v d S$ but that $\operatorname{PvdS}$ is not required for activity (Fig. 6). Our results also suggest that both the reg $A B$ operon and PvdS are required for optimal aerobic activity of the $\operatorname{reg} A B \mathrm{P} 1$ promoter.

Recent evidence has suggested that a regulatory effect of PvdS on regAB may be mediated through PtxR (Hamood et al., 1996). Vasil et al. (1998) also demonstrated that PvdS regulates $p t x R$, and the regulatory cascade postulated by this group suggests that Fur regulates the expression of $p v d S$ in response to iron conditions. PvdS then activates the expression of $p t x R$ and PtxR regulates ETA production either by activation of the $\mathrm{P} 1$ promoter of $\operatorname{reg} A B$, or indirectly by enhanced siderophore production. This model suggests that the regulation of ETA production involves a complex regulatory cascade. We support this hypothesis and believe that the proposed regulation by PtxR acts through the P1 promoter and not the iron-regulated P2, at least in strain PA103 growing under aerobic conditions. The evidence for this hypothesis is fourfold. (1) A putative PtxR binding site is located upstream of the transcriptional start of the P1 promoter (Hamood et al., 1996). (2) A regA-lacZ fusion which contains only the P2 promoter remains tightly iron regulated (Storey et al., 1990), suggesting that PvdSmediated iron regulation is mediated through the P2 promoter. (3) A deletion of the $p t x R$ gene results in decreased expression of siderophore biosynthesis genes and probably culminates in a reduction in intracellular iron levels. This would increase expression of PvdS, as 
Fur would become active under these conditions (Stintzi et al., 1999). (4) Overexpression of PtxR increases ETA synthesis but this production declines over time, reminiscent of P1 promoter activity, suggesting that overexpression of PtxR enhances P1 promoter activity (Colmer \& Hamood, 1999). An alternative possibility is that $P$. aeruginosa Fur may directly act on the $\operatorname{reg} A$ P1 promoter.

In summary, our studies demonstrate that in the chronic lung infections associated with the disease CF, the colonizing $P$. aeruginosa populations transcribe $p v d S$. Furthermore, $p v d S$ transcript accumulation correlates with toxA transcript accumulation, further suggesting that $p v d S$ is involved in the regulatory cascade controlling transcription of the virulence factor ETA. We also show that in the hypertoxigenic $P$. aeruginosa strain PA103, PvdS, a member of the ECF family of alternative sigma factors, is required for approximately $80 \%$ of the ETA production. Moreover, PvdS is essential for the activity of the $\mathrm{P} 2$ promoter of the $\operatorname{reg} A B$ operon. The regulation of the $\operatorname{reg} A B \mathrm{P} 2$ promoter by PvdS may be direct or indirect. Finally we have demonstrated that in strain PA103 grown in an aerobic environment, PvdS along with the $\operatorname{reg} A B$ operon is required for optimal activity from the reg $A B$ P1 promoter. Growth of strain PAO1 in similar conditions does not lead to detectable activity from the regA P1 promoter. This difference could account for the residual ETA activity $(20 \%$ of wild-type) found in a knock-out strain of PA103. Taken together our results indicate that different isolates of $P$. aeruginosa vary in their response to iron concentration; however, on a population basis, PvdS is an important iron-regulated sigma factor that is expressed in the lungs of patients with CF.

\section{ACKNOWLEDGEMENTS}

We thank Drs Vasil, Schweizer and Hynes for the gifts of strains and plasmids, and Leila Donegan for plasmid construction. This research was supported by MRC grant MT15680 to D.G.S.

\section{REFERENCES}

Albus, A. M., Pesci, E. C., Runyen-Janecky, L. J., West, S. E. H. \& Iglewski, B. H. (1997). Vfr controls quorum sensing in Pseudomonas aeruginosa. J Bacteriol 179, 3928-3935.

Ausubel, F. M., Brent, R., Kingston, R. E., Moore, D. D., Seidman, J. G., Smith, J. A. \& Struhl, K. (1991). Current Protocols in Molecular Biology. New York: Wiley.

Barton, H. A., Johnson, Z., Cox, C. D., Vasil, A. I. \& Vasil, M. L. (1996). Ferric uptake regulator mutants of Pseudomonas aeruginosa with distinct alterations in the iron dependent repression of exotoxin A in aerobic and microaerobic environments. Mol Microbiol 21, 1001-1017.

Birnboim, H. C. \& Doly, J. (1979). A rapid alkaline extraction procedure for screening recombinant plasmid DNA. Nucleic Acids Res 7, 1513-1523.

Bodey, G. P., Bolivar, R., Fainstein, V. \& Jadeja, L. (1983). Infections caused by Pseudomonas aeruginosa. Rev Infect Dis 5, 279-313.
Chung, D. W. \& Collier, R. J. (1977). Enzymatically active peptide from the adenosine diphosphate-ribosylating toxin of Pseudomonas aeruginosa. Infect Immun 16, 832-841.

Colmer, J. A. \& Hamood, A. N. (1999). Expression of $p t x R$ and its effect on toxA and $\operatorname{reg} A$ expression during the growth cycle of Pseudomonas aeruginosa strain PAO1. Can J Microbiol 45, 1008-1016.

Cunliffe, H. E., Merriman, T. R. \& Lamont, I. L. (1995). Cloning and characterization of $p v d S$, a gene required for pyoverdine synthesis in Pseudomonas aeruginosa: PvdS is probably an alternative sigma factor. J Bacteriol 177, 2744-2750.

Frank, D. W. \& Iglewski, B. H. (1988). Kinetics of toxA and $\operatorname{reg} A$ mRNA accumulation in Pseudomonas aeruginosa. J Bacteriol 170, 4477-4483.

Frank, D. W., Storey, D. G., Hindahl, M. S. \& Iglewski, B. H. (1989). Differential regulation by iron of $\operatorname{reg} A$ and toxA transcript accumulation in Pseudomonas aeruginosa. J Bacteriol 171, 5304-5313.

Gambello, M. J., Kaye, S. \& Iglewski, B. H. (1993). LasR of Pseudomonas aeruginosa is a transcriptional activator of the alkaline protease gene $(a p r)$ and an enhancer of exotoxin A expression. Infect Immun 61, 1180-1184.

Grant, C. C. R. \& Vasil, M. L. (1986). Analysis of transcription of the exotoxin A gene in Pseudomonas aeruginosa. J Bacteriol 168, 1112-1119.

Haas, B., Kraut, J., Marks, J., Zanker, S. C. \& Castignetti, D. (1991). Siderophore presence in sputa of cystic fibrosis patients. Infect Immun 59, 3997-4000.

Hamood, A. N., Colmer, J. A., Ochsner, U. A. \& Vasil, M. L. (1996). Isolation and characterization of a Pseudomonas aeruginosa gene, $p t x R$, which positively regulates exotoxin A production. Mol Microbiol 21, 97-110.

Hedström, R. C., Funk, C. R., Kaper, J. B., Pavlovskis, O. R. \& Galloway, D. R. (1986). Cloning of a gene involved in regulation of exotoxin A expression in Pseudomonas aeruginosa. Infect Immun 51, 37-42.

Holloway, B. W., Krishnapillai, V. \& Morgan, A. F. (1979). Chromosomal genetics of Pseudomonas. Microbiol Rev 43, 73-102.

Hynes, M. F., Quandt, J., O'Connell, M. P. \& Puhler, A. (1989). Direct selection for curing and deletion of Rhizobium plasmids using transposons carrying the Bacillus subtilus sacB gene. Gene 78, 111-120.

Iglewski, B. H. \& Kabat, D. (1975). NAD-dependent inhibition of protein synthesis by Pseudomonas aeruginosa toxin. Proc Natl Acad Sci US A 72, 2284-2288.

Lamont, I. L., Beare, P. A., Ochsner, U., Vasil, A. I. \& Vasil, M. L. (2002). Siderophore-mediated signaling regulates virulence factor production in Pseudomonas aeruginosa. Proc Natl Acad Sci U S A 99, 7072-7077.

Leoni, L., Ciervo, A, Orsi, N. \& Visca, P. (1996). Iron-regulated transcription of the $p v d A$ gene in Pseudomonas aeruginosa: effect of Fur and PvdS on promoter activity. J Bacteriol 178, 2299-2313.

Leoni, L., Orsi, N., de Lorenzo, V. \& Visca, P. (2000). Functional analysis of PvdS, an iron starvation sigma factor of Pseudomonas aeruginosa. J Bacteriol 182, 1481-1491.

Liu, P. V. (1966). The roles of various fractions of Pseudomonas aeruginosa in its pathogenesis. III. Identity of the lethal toxins produced in vitro and in vivo. J Infect Dis 116, 481-489.

Liu, P. V. (1973). Exotoxins of Pseudomonas aeruginosa. I. Factors that influence the production of exotoxin A. J Infect Dis 128, 506-513. 
Liu, P. V. (1974). Extracellular toxins of Pseudomonas aeruginosa. J Infect Dis S130, S94-S99.

Lory, S. (1986). Effect of iron on accumulation of exotoxin Aspecific mRNA in Pseudomonas aeruginosa. J Bacteriol 168, 1451-1456.

Merriman, T. R., Merriman, M. E. \& Lamont, I. L. (1995). Nucleotide sequence of $p v d D$, a pyoverdine biosynthetic gene from Pseudomonas aeruginosa: PvdD has similarity to peptide synthetases. J Bacteriol 177, 252-258.

Miller, J. H. (1972). Experiments in Molecular Genetics. Cold Spring Harbor, NY: Cold Spring Harbor Laboratory.

Miyazaki, H., Kato, H., Nakazawa, T. \& Tsuda, M. (1995). A positive regulatory gene, $p v d S$, for expression of pyoverdin biosynthetic genes in Pseudomonas aeruginosa PAO. Mol Gen Genet 248, 17-24.

Ochsner, U. A., Vasil, A. I. \& Vasil, M. L. (1995). Role of the ferric uptake regulator of Pseudomonas aeruginosa in the regulation of siderophores and exotoxin A expression: purification and activity on iron-regulated promoters. J Bacteriol 177, 7194-7201.

Ochsner, U. A., Johnson, Z., Lamont, I. L., Cunliffe, H. E. \& Vasil, M. L. (1996). Exotoxin A production in Pseudomonas aeruginosa requires the iron-regulated $p v d S$ gene encoding an alternative sigma factor. Mol Microbiol 21, 1019-1028.

Prentki, P. \& Krisch, H. M. (1984). In vitro insertional mutagenesis with a selectable DNA fragment. Gene 29, 303-313.

Prince, R. W., Storey, D. G., Vasil, A. I. \& Vasil, M. L. (1991). Regulation of toxA and $\operatorname{reg} A$ by the E. coli fur gene and identification of a Fur homologue in Pseudomonas aeruginosa PA103 and PAO1. Mol Microbiol 5, 2823-2831.

Quandt, J. \& Hynes, M. F. (1993). Versatile suicide vectors which allow direct selection for gene replacement in gram-negative bacteria. Gene 127, 15-21.

Raivio, T. L., Ujack, E. E., Rabin, H. R. \& Storey, D. G. (1994). An association between transcript levels of the Pseudomonas aeruginosa $\operatorname{reg} A, \operatorname{reg} B$ and $\operatorname{tox} A$ genes in the sputa of cystic fibrosis patients. Infect Immun 62, 3506-3514.

Raivio, T. L., Hoeffer, D., Prince, R. W., Vasil, M. L. \& Storey, D. G. (1996). Linker insertion scanning of reg $A$, an activator of exotoxin A production in Pseudomonas aeruginosa. Mol Microbiol 22, 239-254.

Rombel, I. T., McMorran, B. J. \& Lamont, I. L. (1995). Identification of a DNA sequence motif required for expression of ironregulated genes in pseudomonads. Mol Gen Genet 246, 519-528.

Sambrook, J., Fritsch, E. F. \& Maniatis, T. (1989). Molecular Cloning: a Laboratory Manual, 2nd edn. Cold Spring Harbor, NY : Cold Spring Harbor Laboratory.

Schweizer, H. P. (1992). Allelic exchange in Pseudomonas aeruginosa using novel ColE1-type vectors and a family of cassettes containing a portable oriT and the counter-selectable Bacillus subtilis sacB marker Mol Microbiol 6, 1195-1204.

Schweizer, H.P. (1993a). Small broad-host-range gentamycin resistance cassettes for site-specific insertion and deletion mutagenesis. Biotechniques 15, 831-834.

Schweizer, H. P. (1993b). Two plasmids, X1918 and Z1918, for easy recovery of the $x y l E$ and lacZ reporter genes. Gene 134, 89-91.

Smith, A. W. \& Iglewski, B. H. (1989). Transformation of Pseudomonas aeruginosa by electroporation. Nucleic Acids Res 17, 10509.
Stintzi, A., Johnson, Z., Stonehouse, M., Ochsner, U., Meyer, J. M., Vasil, M. L. \& Poole, K. (1999). The $p v c$ gene cluster of Pseudomonas aeruginosa: role in synthesis of the pyoverdine chromophore and regulation by PtxR and PvdS. J Bacteriol 181, 4118-4124.

Storey, D. G., Frank, D. W., Farinha, M. A., Kropinski, A. M. \& Iglewski, B. H. (1990). Multiple promoters control the regulation of the Pseudomonas aeruginosa regA gene. Mol Microbiol 4, 499-503.

Storey, D. G., Raivio, T. L., Frank, D. W., Wick, M. J., Kaye, S. \& Iglewski, B. H. (1991). Effect of regB on expression from the P1 and $\mathrm{P} 2$ promoters of the Pseudomonas aeruginosa reg $A B$ operon. J Bacteriol 173, 6088-6094.

Storey, D. G., Ujack, E. E. \& Rabin, H. R. (1992). Population transcript accumulation of Pseudomonas aeruginosa exotoxin A and elastase in sputa from patients with cystic fibrosis. Infect Immun 60, 4687-4694.

Storey, D. G., Ujack, E. E., Mitchell, I. \& Rabin, H. R. (1997). Positive correlation of algD transcription to las $B$ and $\operatorname{las} A$ transcription by populations of Pseudomonas aeruginosa in the lungs of patients with cystic fibrosis. Infect Immun 65, 4061-4067.

Storey, D. G., Ujack, E. E., Rabin, H. R. \& Mitchell, I. (1998). Pseudomonas aeruginosa las $R$ transcription correlates with the transcription of las $A, \operatorname{las} B$ and tox $A$ in chronic lung infections associated with cystic fibrosis. Infect Immun 66, 2521-2528.

Swanson, B. L., Colmer, J. A. \& Hamood, A. N. (1999). The Pseudomonas aeruginosa exotoxin A regulatory gene, ptxS: evidence for negative autoregulation. J Bacteriol 181, 4890-4895.

Vasil, M. L. \& Ochsner, U. A. (1999). The response of Pseudomonas aeruginosa to iron: genetics, biochemistry and virulence. Mol Microbiol 34, 399-413.

Vasil, M. L., Ochsner, U. A., Johnson, Z., Colmer, J. A. \& Hamood, A. N. (1998). The Fur-regulated gene encoding the alternative sigma factor PvdS is required for iron-dependent expression of the LysR-type regulator PtxR in Pseudomonas aeruginosa. J Bacteriol 180, 6784-6788.

West, S. E. H., Sample, A. K. \& Runyen-Janecky, L. J. (1994). The $v f r$ gene product, required for Pseudomonas aeruginosa exotoxin A and protease production, belongs to the cyclic AMP receptor protein family. J Bacteriol 176, 7532-7542.

Wick, M. J., Frank, D. W., Storey, D. G. \& Iglewski, B. H. (1990). Identification of $\operatorname{reg} B$, a gene required for optimal exotoxin $\mathrm{A}$ yields in Pseudomonas aeruginosa. Mol Microbiol 4, 489-497.

Wilson, M. J. \& Lamont, I. L. (2000). Characterization of an ECF sigma factor protein from Pseudomonas aeruginosa. Biochem Biophys Res Commun 273, 578-583.

Wilson, M. J., McMorran, B. J. \& Lamont, I. L. (2001). Analysis of promoters recognized by $\mathrm{PvdS}$, an extracytoplasmic-function sigma factor protein from Pseudomonas aeruginosa. J Bacteriol 183, 2151-2155.

Xiong, Y.-Q., Vasil, M. L., Johnson, Z., Ochsner, U. A. \& Bayer, A. S. (2000). The oxygen- and iron-dependent sigma factor $p v d S$ of Pseudomonas aeruginosa is an important virulence factor in experimental infective endocarditis. J Infect Dis 181, 1020-1026.

Received 3 June 2002; accepted 20 June 2002. 